\title{
Escenario de digitalización para el sistema portuario español
}

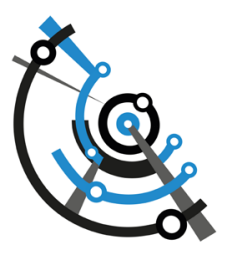

\section{- Nicoletta González-Cancelas}

Departamento de Ingeniería Civil, Transportes, Universidad Politécnica de Madrid, España. https://orcid.org/oooo-0o01-7167-1563

\section{Beatriz Molina Serrano}

Departamento de Ingeniería Civil, Transportes, Universidad Politécnica de Madrid, España. https://orcid.org/oooo-0002-7832-9573

\section{Magdalena Esteban-Infantes}

Departamento de Ingeniería Civil, Transportes, Universidad Politécnica de Madrid, España. https://orcid.org/oooo-0003-2379-5849

\section{Francisco Soler-Flores}

Facultad de Educación, Universidad de La Rioja (UNIR), España. https://orcid.org/oooo-0002-1636-834X

\section{Alberto Camarero Orive}

Departamento de Ingeniería Civil, Transportes, Universidad Politécnica de Madrid, España. ORCID: 0000-0002-0614-8714

Recibido: 24 de junio de 2019. Aceptado: 31 de julio de 2019.

\section{Resumen}

Actualmente, nos encontramos inmersos en la cuarta revolución industrial o industria 4.0. Los puertos están viviendo un proceso de digitalización, similar al de otros sectores industriales y económicos. El objetivo es la automatización para hacer más eficiente y predictiva la cadena de suministro, persiguiendo también la intermodalidad y desarrollar una gestión más sostenible para reducir la contaminación y ganar flexibilidad y agilidad. Sin embargo, este proceso no está exento de complejidad y problemas que dificultan su implantación en el caso español. Es por ello que es papel de los expertos es realizar un análisis de la situación actual, reflexionando sobre las posibilidades y los riesgos que presenta en nuestro país la digitalización del sector portuario. Para llevar a cabo dicho análisis se recurrirá a un panel DELPHI cuyo resultado final será 
la realización de un matriz DAFO. En el artículo se recogen los resultados obtenidos tras el estudio realizado.

Palabras clave: Digitalización. Sistema portuario español. Panel DELPHI. Matriz DAFO. Automatización.

\title{
Digitization stage for Spanish Port System
}

\begin{abstract}
Currently, we are immersed in the fourth industrial revolution or industry 4.0. The ports are experiencing a process of digitalization, similar to that of other industrial and economic sectors. The objective is automation to make the supply chain more efficient and predictive, also pursuing intermodality and developing a more sustainable management to reduce pollution and gain flexibility and agility. However, this process is not without complexity and problems that make it difficult to implement in the Spanish case. That is why it is the experts' role to carry out an analysis of the current situation, reflecting on the possibilities and risks that the digitization of the port sector presents in our country. To carry out this analysis we will use a DELPHI panel whose final result will be the realization of a SWOT matrix. In the article the results obtained after the study are collected.
\end{abstract}

Keywords: Digitization. Spanish port system. DELPHI panel. SWOT matrix. Automation. Palavras-chave: Digitalização Sistema portuário espanhol. Painel DELPHI. Matriz DAFO. Automação.

\section{Introducción}

En cualquiera de los sectores productivos, la transformación digital es muy importante en el mundo de los negocios. Dentro del transporte marítimo, los puertos y la logística sirven para ejemplificar dichos desarrollos, dado que, como actores de las cadenas de suministro mundiales, los puertos marítimos se ven particularmente afectados por el cambio tecnológico. Por otro lado, debido a los altos requisitos en el sector logístico, por ejemplo, con respecto a los costes, la eficiencia, la seguridad y la sostenibilidad, la innovación digital es esencial para mantener la competitividad (Haraldson, 2015).

Los puertos modernos desempeñan un papel importante para garantizar flujos de carga eficientes y seguros en las redes logísticas mundiales. Además, ofrecen varios tipos de servicios de logística de valor agregado que permiten, por ejemplo, facilitar las estrategias de aplazamiento de las cadenas de suministro globales. De ahí que la eficiencia y la seguridad de los flujos de carga relacionados dependen en gran medida de los flujos de información asociados.

Desde el inicio de la contenedorización en la década de 1960, la adopción de la tecnología de la información y los sistemas de información se ha convertido en un factor de éxito indispensable para la competitividad de los puertos, facilitando la comunicación y la toma de decisiones en aras de mejorar la visibilidad, productividad, eficiencia, y seguridad en los procedimientos portuarios que se ven afectados por diversas condiciones (Panayides y Song, 2013). Además, es también cada vez más importante una mejor integración de los organismos gubernamentales para estandarizar y armonizar los trámites de información.

La digitalización está llevando a la industria marítima más allá de sus límites tradicionales y brinda muchas nuevas oportunidades para mejorar la productividad, la 
eficiencia y la sostenibilidad de la logística (Cimino et al., 2016). El concepto de puertos inteligentes, por ejemplo, apunta a adoptar tecnologías de información modernas que permitan una mejor planificación y administración dentro y entre los puertos. Las necesidades más urgentes de la digitalización son inversiones en tecnología y cooperaciones para promover un intercambio de información y una mejor coordinación y colaboración, a menudo considerados como un obstáculo en entornos altamente competitivos. Además de estas nuevas oportunidades, surgen importantes problemas y, entre ellos, problemas económicos.

El rol de los puertos ha cambiado drásticamente de su función tradicional de conexión del mar y la tierra mediante las operaciones de carga y descarga, a su nueva función como parte esencial de las redes logísticas globales que gestionan el flujo de carga y ofrecen servicios logísticos de valor agregado de una manera eficiente y efectiva, así como segura y respetuosa con el medio ambiente. Es por ello que, debido a la importancia de su papel para lograr una ventaja competitiva, en las últimas décadas se han adoptado una gran cantidad de sistemas y tecnologías de información en las operaciones portuarias, que han permitido que cada vez se realicen más transacciones electrónicas (Heilig y Voß, 2017a). Sin embargo, pese a que dichos desarrollos han conducido a un alto grado de digitalización y automatización, especialmente en terminales de contenedores, todavía existe un considerable potencial de mejora.

Por ello, la fase actual de la transformación digital está muy enfocada a adoptar nuevas tecnologías digitales para medir, monitorear y controlar mejor las operaciones de los puertos permitiendo, por ejemplo, utilizar datos operativos en tiempo real para predecir eventos futuros (Heilig y Voß, 2017b). Este hecho implica un preprocesamiento avanzado y un análisis de datos para extraer información y conocimientos, que se pueden utilizar en la planificación avanzada y en los sistemas de soporte de decisiones. Sin embargo, el éxito de la transformación digital radica no solo en el uso de tecnologías y métodos avanzados, sino en la adaptación de los aspectos organizativos bajo la idea de que "la tecnología digital es un medio, no un fin" (Grasso, Pagola y Zanotti, 2017).

Este documento señala la digitalización como una de las tecnologías centrales del siglo XX. En particular, la digitalización de puertos se está convirtiendo en la dirección del desarrollo futuro. Por ello, se analiza el estado de la digitalización de puertos y se propone tanto el objetivo de estrategia para el desarrollo de la digitalización de España como los pasos para llevar a cabo dicho objetivo en diferentes períodos. Finalmente, se presentan algunas medidas y sugerencias para el desarrollo de la digitalización de puertos en España, si bien, en general, los sistemas de información ya abarcan casi todas las actividades de logística marítima utilizadas principalmente para adquirir, procesar, intercambiar y analizar datos a un volumen y ritmo cada vez mayores.

\section{La situación en los puertos españoles}

Según indica el Ministerio de Fomento de España (Ministerio de Fomento, 2017) la situación actual de la digitalización portuaria es debida a la persistencia de obstáculos de tipo administrativo, tales como la gran cantidad de documentación necesaria en transporte marítimo que sigue siendo muy superior a la utilizada en transporte terrestre, $y$ debida a obstáculos de tipo económico, dado que la intermodalidad terrestre-marítima intracomunitaria sigue siendo más cara que envíos realizados íntegramente por carretera y dado que la inversión inicial necesaria en este tipo de iniciativas es muy elevada.

Por otro lado, en relación con la mejora en el rendimiento operativo de terminales y la reducción de sus costes asociados, cabe destacar que las terminales se han tenido que ir adaptando al crecimiento de los buques mediante la ampliación de sus capacidades y 
medios mecánicos, al tiempo que han tenido que introducir innovaciones relacionadas con la digitalización y automatización de procesos, similares a las mejoras que se han introducido en otros sectores de producción, tales como el de la automoción

Por tanto, las futuras política de transportes en España deberán considerar e incluir en sus enfoques multidimensionales los retos que presentan los sistemas de transporte que están por venir, así como la inserción de la dimensión digital e informacional, planteando modelos de transporte que respondan equitativamente a sus necesidades en un espacio híbrido en el que se combine lo físico con lo digital o virtual.

En el mundo actual, se habla de Smart, 4.0, digitación, innovación... puesto que con los medios disponibles, es posible cambiar el funcionamiento de todo el sistema mundial. Esta evolución es inevitable, puesto que el avance tecnológico no se puede detener; y puesto que el mundo así lo requiere. Es por ello que ha llegado el momento de hacer (y aplicar) opciones más sostenibles y más orientadas hacia la vida futura, en lugar de obtener ganancias inmediatas.

Dentro de estas tendencias, la innovación juega un papel fundamental. No obstante, éste debe ser un proceso controlado, puesto que implica un cambio cultural, donde hay que recrear los flujos y procesos para generar una innovación abierta. La innovación debe formalizarse e incorporarse a la cultura de la empresa, de forma que los puertos, como industria-empresa, no están ajenos a este proceso. Así, en el entorno portuario, la gestión del modelo $\mathrm{I}+\mathrm{D}+\mathrm{i}$ asociado a la innovación debe ser sistematizado, comprobable, auditado y medible, de forma que, para garantizar el control de las actividades innovadoras se deben desarrollar herramientas de control. No obstante, todavía queda un largo camino dentro del sector portuario para controlar la gestión del cambio de iniciativas a proyectos.

El concepto de puerto 4.0 va ligado al concepto de industria 4.0, que se asocia al fenómeno de una cuarta revolución industrial. Esta cuarta revolución supone un cambio en el desarrollo industrial capaz de generar importantes cambios sociales en los próximos años. Así, se trata de un concepto de industria en el que se hace un uso intensivo de Internet y de tecnología puntera con el fin de desarrollar plantas y procesos industriales más inteligentes y respetuosos con el medio ambiente, lo que implica cadenas de producción rigurosamente comunicadas entre sí y con los mercados.

Puertos del Estado ha generado un plan denominado Plan Puertos 4.0 mediante el cual se aporta un fondo para impulsar la innovación en el sector portuario. El Fondo Puertos 4.0 es un nuevo fondo de compensación interportuario dirigido a promover e incorporar activamente la innovación disruptiva como elemento de competitividad en el sector logístico-portuario tanto público como privado, así como de eficiencia, sostenibilidad, seguridad, etc. Las estrategias para alcanzar los objetivos del plan se basan en complementar la innovación interna con la externa a nivel de sistema portuario y de comunidad portuaria, introducirse en ecosistemas innovadores nacionales e internacionales, disponer de una incubadora de startups corporativa del sector logístico-portuario y acceder a financiación y facilitar la captación de inversión privada para la innovación portuaria.

En definitiva, el objetivo del Plan Puertos 4.0 es impulsar el emprendimiento para fomentar la innovación, articulando y vinculando un ecosistema de innovación externo que esté al servicio del sector logístico-portuario. Con ello, se espera conseguir la consolidación de un tejido de empresas emergentes y nuevas líneas de negocio que desarrollen productos innovadores para el sector logístico-portuario, así como la aplicación práctica de nuevas estrategias de innovación, potenciando la adaptación y transición del sector logístico-portuario a la industria 4.0. 
Puertos del Estado puso en marcha el Fondo Puertos 4.0 en junio de 2019, siendo el mayor fondo de innovación abierto nunca para el ecosistema logístico y portuario español. Nutrido por la aportación de todas las autoridades portuarias de España (correspondiente al $2 \%$ de sus beneficios) y con una cuantía de 25 millones de euros esta "hucha" se destinará a financiar los mejores ideas y proyectos que avancen hacia la digitalización de los puertos españoles. Además de la cadena logística también se busca financiar proyectos en el sector comercial, teniendo en cuenta que este es un eje básico de la actividad portuaria. Asimismo incluye el transporte de mercancías y vehículos, el turismo, la construcción de infraestructuras, etc. todo desde un punto de vista de innovación y digitalización, de cara a avanzar en el smart port o el puerto 4.0.

El programa tendrá una duración de cuatro años y contará con varias convocatorias puesto que la intención de Puertos del Estado es dar continuidad al programa si los resultados son positivos, así como ampliar el actual fondo de 25 millones.

La transformación hacia el puerto 4.0 pasa por abordar medidas para potenciar la eficiencia logística en el ámbito de las infraestructuras, de las operaciones y de la prestación de servicios, así como mejorar a sostenibilidad ambiental y energética, la seguridad y protección, y fomentar la digitalización de procesos y plataformas inteligentes.

Los puertos han jugado desde siempre un papel clave en el desarrollo de la sociedad siendo un elemento fundamental en el desarrollo económico de los distintos países por lo que se sitúan en una posición estratégica a nivel mundial. Los actuales cambios a nivel global motivados por nuevas formas de comunicación, patrones de comportamiento, tecnologías innovadoras, etc. han propiciado que los puertos no puedan quedarse al margen de estas nuevas tendencias; debiendo tomar un papel activo, constituyéndose como un elemento principal de la transformación. Es por todo ello que en el presente artículo se trata de analizar los aspectos más relevantes de la digitalización de los puertos en su afán de transformarse en smart ports o puertos 4.0.

Una solución smart es la que proporciona a la gente mejoras tanto en su ámbito personal como en el colectivo, en relación al territorio donde vive y su entorno (Cervantes et al., 2013). Una solución smart no es lo mismo que una solución digital, pero las tecnologías de la información y comunicación (TIC) son un buen camino para conseguir ese propósito. Para alcanzar dicho objetivo, la digitalización se presenta como una condición sine qua non en el desarrollo de los puertos. En este artículo se analizan también las principales Debilidades, Amenazas, Fortalezas y Oportunidades que se encuentran en el proceso de digitalización de los puertos, definiendo tanto las claves para obtener la máxima eficiencia a través de la digitalización como los elementos en los que ha de sustentarse.

El objetivo final será la elaboración de un mapa de perspectiva global a corto y medio plazo con los elementos más destacados por los expertos, diferenciando entre los elementos que pongan innovación en valor y los que suponen tan sólo imitación en valor.

\section{Tendencias actuales}

\section{Innovación, digital y smart}

Es posible un mundo smart con soluciones innovadoras y digitales. Como ya se indicado, los pilares son la innovación, las soluciones smart y el empoderamiento organizacional a través de la digitalización (Sánchez y Barleta, 2018). 
La innovación en el sector portuario va a requerir en este proceso de la participación de la ciudadanía, del talento de la personas para mejorar la creatividad, prototipar para obtener los mejor haciendo referencia a la acción y el proceso de creación de un modelo de un determinado producto y a las sucesivas pruebas que se hacen con él y tener herramientas para gestionar el cambio que va a producirse en los puertos. Así, las soluciones smart asociadas al industria 4.0 portuaria deben apostar por la simplicidad, la sostenibilidad, y estar focalizadas en la eficiencia, necesitando tener a la gente y el territorio en contacto.

El empoderamiento organizacional a través de la digitalización necesita de una revisión de los procesos portuarios. Así, se necesitará del empleo de la tecnología digital, mejorando las competencias digitales actuales (Sánchez y Mouftier, 2016), si bien, se debe tener en cuenta que aparecerán nuevos roles en el proceso. Este proceso de empoderamiento digital se basa en:

» Tener poder de decisión propio.

» Tener acceso a la información y a los recursos para tomar una decisión apropiada.

» Tener una gama de opciones para elegir.

" Habilidad para ejercer asertividad en la toma de de-cisiones colectivas.

" Tener un pensamiento positivo y la habilidad para hacer cambios.

" Habilidad para aprender y para mejorar su propio poder personal o de grupo.

" Habilidad para cambiar las percepciones por me-dios democráticos.

» Mejorar la autoimagen y superar la estigmatización.

" Participar en un proceso autoiniciado de crecimiento y cambios continuos.

Actualmente, nos encontramos inmersos en la cuarta revolución industrial o industria 4.0 que exige pasar de puertos electrónicos a puertos interconectados. Este hecho va a producir cambios en los modelos de gobernanza portuaria y no sólo porque se prefiera que los puertos sean más públicos o más privados, sino porque la manera de competir y de ser eficiente será diferente y requerirá una adecuación de la gestión. Todos estos procesos de transformación necesitan básicamente los 6 factores para el éxito que se recogen en la Figura 1.
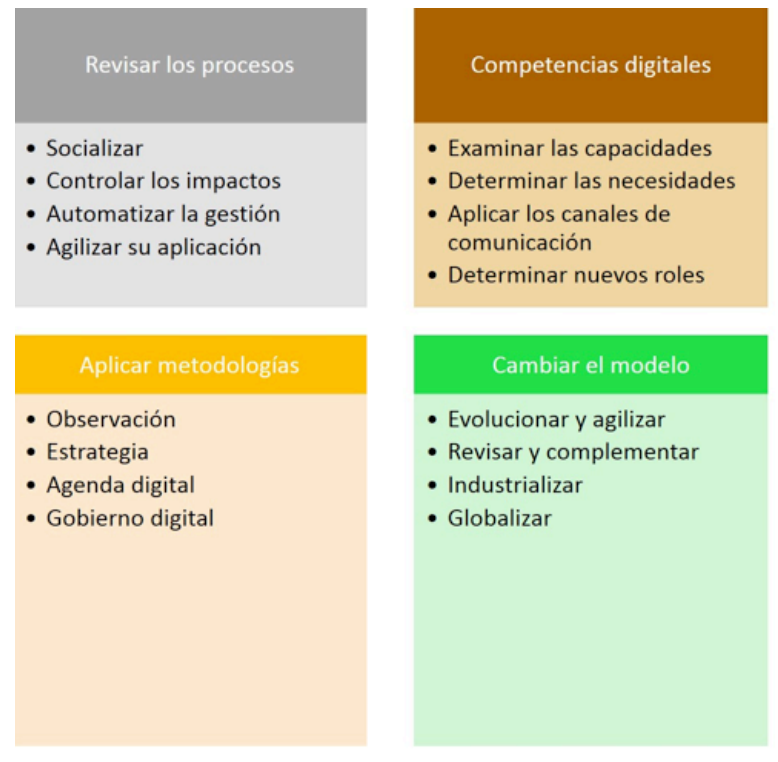

Mejorar la user experience

- Usabilidad

- Accesibilidad

- Simplificación

- Diseño

Usar tecnología
- Big Data \& Analytics
- Robótica (M. Learning \& A.
Vision)
- Impresión 3D
- Movilidad \& Wearables
- Biotecnología
- Soluciones smart
- Realidad Extendida y Virtual
- Redes Sociales y eComercio
- Ciberseguridad

Figura 1. Factores para el éxito. Fuente: Elaboración propia. 


\section{El reto de la digitalización de puertos innovadores y smart}

Los factores a considerar para la generación de puertos digitalizados, innovadores y smart se dividen en factores internos a los puertos y factores externos a los mismos (Sánchez, Palma Barleta y Mouftier, 2017).

Los factores internos se centran más en la eficiencia y, por tanto, suponen un cambio cultural. Entre los más destacados se encuentran:

" Posición legal del sector público, que afecta a la gobernanza y las posibilidades para introducir la innovación

" Compañías y competitividad, en lo relativo a la brecha existente entre los procesos industriales y la excesiva burocratización de las compañías y la administración

» Propiedad del puerto, que complica su gestión en un contexto de necesaria interrelación

Por el contrario, los factores externos están más centrados en el crecimiento y suponen nuevas relaciones, destacando entre ellos los siguientes:

» Logística y la industria: barcos cada vez más grandes que dificultan su amarre y cambian calendarios, previsiones y operativa.

"Economía: la globalización y las tensiones eco-nómicas, así como la difícil situación geopolítica actual

" Comercio: nuevas formas de comercio que han roto los moldes tradicionales

\section{Las herramientas disponibles}

Actualmente el Big Data y el Blockchain son las principales herramienta para crear smart ports, similar a lo ocurrido en el caso de las smart cities (Lee et al., 2018). Los datos se recogen en cualquier lugar y en cualquier momento, lo que representa una espada de doble filo, ya que por un lado permiten a los usuarios ofrecer servicios adaptados a ellos, pero al mismo tiempo, se mueven en el límite entre "búsqueda de datos" e "invasión de la privacidad". No obstante, el Blockchain está considerado a todos los efectos una innovación que en pocos años entrará en nuestras vidas (Davarzani et al., 2016).

El hecho de tener un Puerto 4.0 comprende una serie de ventajas, como por ejemplo tener la mercancía localizada en todo momento, lo que ofrece una mayor posibilidad de control, que se traduce en una mayor eficacia en cuanto a carga-descarga, almacenamiento y futuro traslado dentro del Hinterland (Brouer, Karsten y Pisinger, 2016). Otro ejemplo sería la utilización de vehículos eléctricos para el movimiento de cargas, puesto que este tipo de vehículos contribuye a reducir emisión local de gases de efecto invernadero (Arguedas, Pallotta y Vespe, 2017). Además, desde el punto de vista de ruido y posible vertido de combustibles de maquinaria, desde el punto de vista ambiental y paisajístico, se reduce el impacto que estos factores pueden producir en un entorno tan sensible como es un puerto.

Es obvio pensar que los puertos que poseen un alto desarrollo informático son más competitivos y eficientes que los que tienen ausencia de este. De esta manera, son infraestructuras que podrán trabajar con mayor volumen de tráfico anual, logrando un mayor intercambio de mercancía, y con ello, mayores ingresos. Además, y de manera indirecta, el hecho de que en una zona crezca comercialmente, genera un efecto de "rivalidad" mediante el cual las zonas colindantes también desean crecer, creándose un ambiente en el que se produce un desarrollo positivo, tanto para el consumidor final (quien paga por una determinada mercancía), como para el ámbito regional al tratarse de un punto clave para la economía de la zona. Con la digitalización, los puertos buscan la automatización para hacer más eficiente y predictiva la cadena de suministro (Zaman 
et al., 2017), persiguiendo también la intermodalidad y el desarrollo de una gestión más sostenible que permita reducir la contaminación y ganar flexibilidad y agilidad. Como no podía ser de otra manera, los puertos españoles se han sumado al proceso de digitalización, y poco a poco, las acciones llevadas a cabo les confieren fortalezas que marcan una clara competitividad.

Basado en una revisión sistemática de la literatura, el artículo de Markus Fruth and Frank Teuteberg (Fruth y Teuteberg, 2017) es el artículo más completo sobre revisión de la literatura de digitalización hasta la fecha. Dicho artículo proporciona una visión general del estado actual de la digitalización en la logística marítima, analiza las áreas problemáticas existentes y muestra el potencial de mejora. Los resultados muestran que es esencial capturar el potencial de desarrollo para poder beneficiarse de las ventajas. Sin embargo, el artículo concluye que la investigación aún se encuentra en sus etapas incipientes y faltan trabajos teóricos y empíricos, así como enfoques explicativos para las poder realizar recomendaciones apropiadas para la acción y la reestructuración.

\section{Metodología}

El análisis que aquí se presenta se ha realizado con una metodología DAFO o análisis FODA, a través del estudio interno de las debilidades y fortalezas y del estudio externo de las amenazas y oportunidades de la transformación digital de los puertos, destacando las principales ideas de cada aspecto y dando una breve descripción de las mismas.

Las debilidades, de carácter interno, hacen referencia a las carencias y limitaciones desfavorables, desde el punto de vista de la autoridad portuaria. Por el contrario, las fortalezas describen las características y habilidades favorables propias. Por el contrario, desde el punto de vista externo se consideran las amenazas (no dependen del puerto) y las oportunidades.

La matriz DAFO permite a los gestores tener una idea general de las dificultades, riesgos y beneficios que aporta la digitalización logística y la información tecnológica al sector portuario y, por ende, a la sociedad.

La elección de una metodología DAFO se basa en los bajos costes que presenta, puesto que dicha herramienta puede ser implementada por cualquier tipo de negocio sin que suponga costes asociados, no necesitando de grandes inversiones ni recursos para realizar una primera foto de la situación actual de la digitalización en los puertos. No hay que olvidar que el DAFO es un método de tipo preventivo, si bien el énfasis está puesto en el conocimiento de las ventajas y las desventajas de las compañías, al minimizar los riesgos promueve prácticas preventivas dentro de su filosofía corporativa.

El enlace de aspectos internos y externos es un aliciente para los procesos estratégicos. Nada puede funcionar de forma aislada ni independiente, pues más tarde o más temprano quedará evidenciado su nexo con el resto de las partes, por eso se ha utilizado esta metodología para integrar los procesos.

Para la determinación de la matriz DAFO se ha desarrollado un panel de expertos que han sido los responsables de su confección, para lo cual ha sido necesario hacer una foto del escenario actual sobre la digitalización.

Se ha de tener en cuenta que en este proceso el análisis DAFO permite realizar un análisis de la digitalización del sector portuario en un momento determinado. Por 
tanto, no se trata de un análisis a proyección futura, si bien se pueden deducir ideas del mismo aunque no basta por sí solo para responder a la situación futura.

La metodología adoptada se ajusta al esquema de la Figura 2.

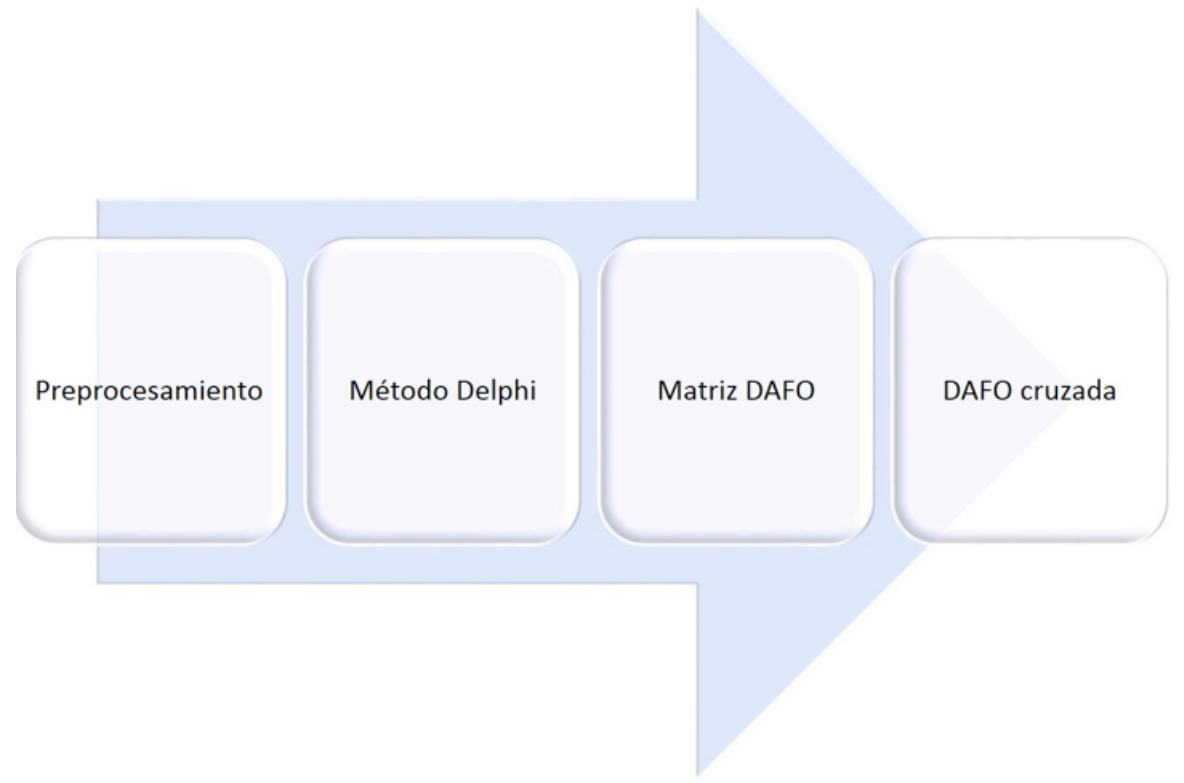

Figura 2. Esquema metodológico. Fuente: Elaboración propia.

El detalle de la metodología empleada es el que se recoge en el Cuadro 1, reflejando fielmente los pasos que se realizaron entre enero y diciembre de 2018, cuando se finalizó la investigación.

Cuadro 1. Descripción de la metodología seguida en el estudio. Fuente: Elaboración propia

\begin{tabular}{|l|l|l|}
\hline Proceso & Paso & Descripción \\
\hline Preprocesamiento & $\begin{array}{l}\text { Paso 1: Selección de } \\
\text { líderes del proceso }\end{array}$ & $\begin{array}{l}\text { Se seleccionan s líderes para el desarrollo y organización de los paneles de } \\
\text { expertos y las etapas de la matriz DAFO. } \\
\text { Los líderes corresponde a las iniciales: NGC, BMS, ACO, MEI, FSF }\end{array}$ \\
\cline { 2 - 3 } & $\begin{array}{l}\text { Paso 2: Bechmarking } \\
\text { internacional }\end{array}$ & $\begin{array}{l}\text { El benchmarking se está convirtiendo en un instrumento central para mejorar } \\
\text { el desempeño del sector público. Este desarrollo refleja que, en las condiciones } \\
\text { adecuadas, la comparación puede ser un importante impulsor del rendimiento. } \\
\text { La evaluación comparativa es un instrumento importante para comparar y } \\
\text { evaluar el desempeño de una manera más objetiva. Se pretende: } \\
\text { - Crear una presión sostenida para mejorar. } \\
\text { - Exponer áreas donde se necesita mejorar y revelar los problemas subyacentes } \\
\text { de una organización o un grupo de organizaciones. } \\
\text { - Identifique procesos superiores para adoptarlos y obtener una mejor } \\
\text { comprensión de las mejores prácticas. } \\
\text { - Centrarse en los vínculos entre procesos y resultados. } \\
\text { - Probar si la mejora ha sido exitosa. } \\
\text { Los } 5 \text { líderes son los encargados de recoger todas las referencias internacionales } \\
\text { yorganizarlas para trabajar en equipos con expertos del sector }\end{array}$ \\
\cline { 2 - 3 } & $\begin{array}{l}\text { Paso 3: Lista de } \\
\text { verificación }\end{array}$ & $\begin{array}{l}\text { Todos los elementos que afectan a la digitalización de puertos en España deben } \\
\text { reunirse. Serán los líderes quienes seleccionarán los elementos más relevantes } \\
\text { con los que se trabajarán en el panel de expertos }\end{array}$ \\
\hline
\end{tabular}




\begin{tabular}{|c|c|c|}
\hline Proceso & Paso & Descripción \\
\hline \multirow[t]{6}{*}{ Método Delphi } & $\begin{array}{l}\text { Paso 1: Eligir } \\
\text { un facilitador o líder }\end{array}$ & $\begin{array}{l}\text { Elegir tu facilitador o líder para cada uno de los s equipos de trabajo, que serán } \\
\text { personas neutrales dentro de cada equipo. Es útil tener a alguien que esté } \\
\text { familiarizado con la investigación y la recopilación de datos }\end{array}$ \\
\hline & $\begin{array}{l}\text { Paso 2: Identifiqcar a sus } \\
\text { expertos }\end{array}$ & $\begin{array}{l}\text { La técnica Delphi se basa en un panel de expertos. Un experto es, cualquier } \\
\text { persona con conocimientos relevantes y experiencia en un tema en particular } \\
\text { Cada uno de los líderes va a hacer frente a un equipo de modo que se dispondrá } \\
\text { de } 5 \text { equipos que trabajan de forma independiente en el desarrollo de } 5 \text { matrices } \\
\text { dafo paralelas hasta llegar a la matriz final de consenso. Es difícil trabajar con } \\
\text { muchas personas y ponerlas de acuerdo así que se ha propuesto trabajar en } \\
\text { indicio en } 5 \text { grupos. }\end{array}$ \\
\hline & $\begin{array}{l}\text { Paso 3: Definición del } \\
\text { problema }\end{array}$ & $\begin{array}{l}\text { ¿Cuál es el problema o problema que busca comprender? Los expertos } \\
\text { necesitan saber qué problema están comentando, así que asegúrese de } \\
\text { proporcionar una definición precisa y completa. En este caso versa sobre la } \\
\text { situación actual de la digitalización en el sistema portuario español }\end{array}$ \\
\hline & $\begin{array}{l}\text { Paso 4: Preguntas de } \\
\text { la primera ronda por } \\
\text { equipos }\end{array}$ & $\begin{array}{l}\text { Se desarrollan preguntas generales para obtener una amplia comprensión de } \\
\text { la opinión de los expertos sobre eventos futuros. Las preguntas pueden salir } \\
\text { en forma de cuestionario o encuesta. Recopile y resuma las respuestas, elimine } \\
\text { cualquier material irrelevante y busque puntos de vista comunes. En este caso } \\
\text { es un cuestionario que se basa en la lista de chequeo previa que desarrollan los } \\
\text { líderes de equipo }\end{array}$ \\
\hline & $\begin{array}{l}\text { Paso 5: Cruce de } \\
\text { matrices DAFO previas } \\
\text { por equipos }\end{array}$ & $\begin{array}{l}\text { El líder de cada equipo harán llegar al resto de los equipos su primera DAFO } \\
\text { para que el resto de los equipos estudien los resultados cruzados }\end{array}$ \\
\hline & $\begin{array}{l}\text { Paso 6: Preguntas de la } \\
\text { segunda ronda }\end{array}$ & $\begin{array}{l}\text { Sobre la base de las respuestas a las primeras preguntas, las siguientes } \\
\text { preguntas deben profundizar en el tema para aclarar problemas específicos. } \\
\text { Estas preguntas también pueden enviarse en forma de cuestionario o encuesta. } \\
\text { Nuevamente, se compila y se resumen los resultados, se elimina cualquier } \\
\text { material irrelevante y se busca el terreno común en la búsqueda de construir } \\
\text { consenso. } \\
\text { El cuestionario final apunta a enfocarse en apoyar la toma de decisiones. } \\
\text { Enfocarse en las áreas de acuerdo. ¿En qué están de acuerdo todos los expertos? }\end{array}$ \\
\hline Matriz DAFO & Paso 1: Matriz DAFO & $\begin{array}{l}\text { Después de esta ronda de preguntas, se espera que los expertos alcancen un } \\
\text { consenso y se tenga una visión de los eventos futuros. Se analizan los hallazgos } \\
\text { y establecemos planes para hacer frente a los riesgos y oportunidades futuras } \\
\text { para su proyecto. }\end{array}$ \\
\hline DAFO cruzada & Paso 1: DAFO cruzada & $\begin{array}{l}\text { Una DAFO Cruzada es una derivación del análisis DAFO, en el que los factores } \\
\text { se combinan buscando la potencialización (cuando ambos son positivos o } \\
\text { negativos) entre ellos o la anulación (cuando uno es positivo y otro negativo). }\end{array}$ \\
\hline
\end{tabular}

\section{Resultados}

A través del panel Delphi, los expertos agruparon en una matriz DAFO los elementos de digitalización en el sistema portuario español. La selección de expertos fue muy rigurosa con objeto de que los resultados obtenidos se adhieran a la realidad del sistema portuario español. El elenco de expertos estuvo compuesto tal y como se indica en la Figura 3. 

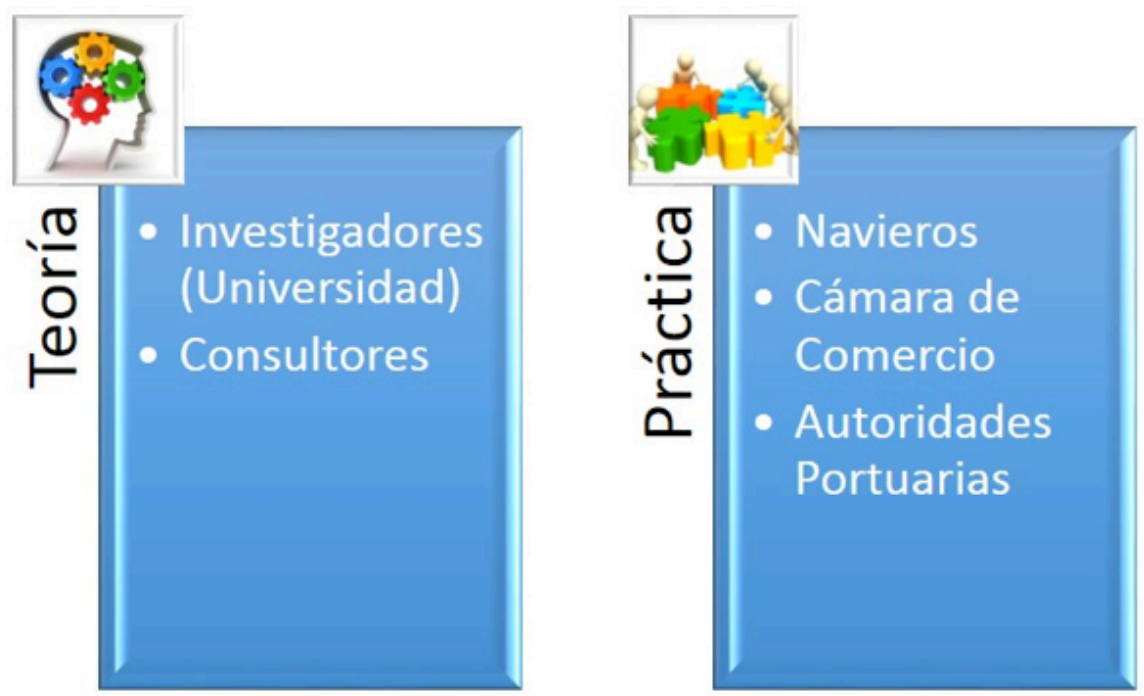

Figura 3. Composición del panel de expertos. Fuente: Elaboración propia.

Tal y como se describe en el Cuadro 1, cada grupo de expertos, guiados por un líder, participó en dos rondas de cuestionarios. Cada grupo estuvo formado por equipos homogéneos en número ( 6 expertos por grupo) y heterogéneos de su composición (ver Figura 3). La selección de los expertos fue minuciosa y se les exigió un compromiso expreso con el proyecto para poder acometerlo en el plazo establecido.

Acorde al tema a tratar en el trabajo y su relación con las nuevas tecnologías, toda la información se distribuyó de manera telemática, disponiendo de toda la información en la nube, en un espacio abierto a los participantes. Asimismo, las reuniones se mantuvieron a través de un software popular de uso común que permite comunicaciones de texto, voz y vídeo sobre Internet.

Cabe señalar que el consenso fue la nota predominante en cada uno de los grupos (intragrupo) y en las relaciones intergrupales, lo que permitió llegar al consenso en la segunda ronda. La mayor dificultad se presentó al agrupar la gran cantidad de subelementos en cada uno de los análisis internos y externos para la generación de la DAFO, si bien los expertos tenían muy claro que elementos participantes en cada uno de los bloques de la DAFO. A continuación se recogen dichos agrupamientos a través del análisis interno y externo de factores.

\section{Análisis interno}

Las fortalezas y debilidades del tema de la digitalización se refieren a la delicada relación entre las nuevas tecnologías al servicio de las infraestructuras y los usuarios de estas últimas. Su definición se basó en un análisis interno de la situación portuaria española.

\section{Debilidades}

Las debilidades son puntos negativos que tendrá la digitalización del puerto, es decir, problemas o situaciones que surgirán y son perjudiciales para el puerto o el sistema portuario.

\section{D.1 Altos costes de desarrollo e implementación de las nuevas tecnologías}

La digitalización y automatización de los procesos en las terminales portuarias crea una necesidad de personal suficientemente capacitado para el uso de las nuevas tecnologías 
o para la manipulación de mercancías, sobre todo cuando se trata de mercancías especiales o de terminales de contenedores. En este sentido, muchos puestos de trabajos quedarían infracualificados o la empresa podría perder control, ya que el personal existente debería reciclarse o sería necesaria la creación de un nuevo profesional técnico y especializado mediante títulos que proporcionan las aptitudes y competencias requeridas acordes a las nuevas tecnologías implantadas.

\section{D.2 Capital humano}

Los trabajadores manuales se encuentran bajo la incidencia directa de la automatización de procesos inherente a la digitalización. Además, se presenta el problema de la inseguridad por la formación que hace que esta falta de información de la plantilla derive comúnmente en el rechazo a la formación en materia digital. Se trata de un problema focalizado en los trabajadores que realizan tareas fundamentalmente manuales o mecánicas, puesto que la implantación de un sistema de digitalización hará que determinadas funciones no sean necesariamente desempeñadas por personas. Estas personas se convertirían en personal especializado que dejará de trabajar, lo que supondrá una pérdida de puestos de trabajo que repercutirá, en mayor o menor medida, en la sociedad, economía... Al implantar nuevos procesos, el personal del puerto debe recibir una formación específica para poder conocer en profundidad los sistemas, puesto que el desconocimiento de los mismos frenaría el proceso de digitalización.

\section{D.3 Vulnerabilidades o errores de regresión. Seguridad e integridad de la empre- sa para transformarse al concepto 4.0.}

La digitalización de los puertos hace posible la adopción de sistemas que aumentan la eficacia y transparencia de los distintos agentes implicados y eliminan la necesidad de documentos de papel, lo cual redunda, en general, en un aumento de la seguridad. Sin embargo, ciertos sistemas de blockchain pueden introducir vulnerabilidades o errores de regresión por defecto a medida que se van haciendo más complejos o a medida que la información es encriptada. Por este motivo, contar con una forma consistente de gestionar y administrar la seguridad del puerto es clave para asegurar su funcionalidad y buena implementación. La inutilización de parte de los servidores que componen el sistema debido a cibera-taques, pueden generar problemas en las operaciones portuarias o afectar también a alguno de sus clientes, de forma que la recuperación de estos sistemas es lenta y lleva aparejadas importantes pérdidas económicas.

\section{D.4 Carente planificación estratégica}

Pese a vivir en un mundo globalizado y con grandes avances tecnológicos, muchos de los puertos tienen un conocimiento insuficiente del catálogo de posibles soluciones de digitalización que puede ofrecerles el mercado. Además, existe una falta de información acerca de cómo funciona, dónde se aplica o qué ventajas puede ofrecer la digitalización. Hablar de digitalización divide el problema en dos cuestiones fundamentales: la primera relativa a la no percepción de la digitalización y la segunda relativa a la no accesibilidad a ella. Esta circunstancia se produce también, a cierta escala, en los puertos, si bien el proceso de digitalización no debe influir en el desarrollo de la actividad portuaria. Para ello es necesaria una planificación ad hoc en cada puerto que le permita cubrir las necesidades específicas de cada instalación y proceso. La instauración de nuevos sistemas requiere de una buena planificación ante las adversidades que se puedan plantear puesto que muchos de los problemas que se puedan presentar no contarán con precedentes dentro del Sistema Portuario español, por lo que la falta de experiencia de los operadores puede suponer una incapacidad de reacción ante estos posibles problemas adversos. 


\section{D.5 Efectos del cambio en la operativa}

La digitalización de los puertos haría también que muchos puestos de trabajo se quedaran obsoletos o fueran innecesarios debido, por ejemplo, a la implementación de los recientes y novedosos sistemas de carga y descarga controlados mediante monitores. Este hecho haría que el número de trabajadores descienda significativamente y, en consecuencia, estaría en una mayor o menor medida garantizado el rechazo del gremio de estibadores a la modernización. En esta situación puede afirmarse que, aunque a largo plazo la implantación de estos sistemas sería, sin lugar a dudas, un éxito, el proceso de cambio puede verse dificultado por las discrepancias creadas entre este gremio y las distintas terminales operadoras, lo que podría ocasionar pérdidas debidas a huelgas o piquetes hasta llegar a un acuerdo fijo entre las dos partes.

\section{D.6 Control y monitoreo del sistema digital}

El hecho de la digitalización impone una dependencia de un sistema digital fiable, estable y seguro, ya que toda la operación del puerto dependerá del mismo. Asimismo debe estar controlado y monitoreado para detectar cualquier fallo, así como protegido frente a agentes (internos o externos) que puedan producir irregularidades.

\section{D.7 Problema de la heterogeneidad de las aplicaciones}

La proliferación de la digitalización presenta el problema de la heterogeneidad de las aplicaciones que se usan globalmente. Un consenso en el estándar de utilización de los formatos abiertos digitales supone una clara debilidad. Para poder proceder a la digitalización de los puertos, en primer lugar se debe globalizar todo el proceso del transporte, es decir, conseguir que todos los países, barcos y mercancías utilicen un sistema común para conseguir una plena coordinación de los sistemas.

\section{Fortalezas}

A continuación, se exponen las principales fortalezas que adquieren los puertos españoles a raíz de las actuaciones de digitalización (implantadas o en fase de desarrollo).

\section{F.1 Ser referente en el sector}

La digitalización supone un incremento de la potencia industrial y de los servicios portuarios diferenciadores lo que permite convertir al puerto en un nodo principal de desarrollo. Asimismo, aporta una distinción dentro del sector. El Sistema Portuario Español es maduro y moderno y los puertos conectados a la red constituyen el marco logístico que permite las transformaciones necesarias en las secciones Smart, Green y Synchromodality. La evolución hacia puertos más eficientes, más funcionales, más avanzados tecnológicamente y más seguros es un desafío constante en los últimos años. Durante años, se han desarrollado herramientas y soluciones que contribuyen a la optimización segura de muchos procesos portuarios, entre los que se encuentran los sistemas de amarre, que permiten controlar y facilitar las operaciones de aproximación y acoplamiento de los barcos gracias a la tecnología láser, proporcionando lecturas muy precisas de velocidad y distancia.

\section{F.2 Consolidación de los puertos españoles como conexión conectores de la actividad portuaria}

Se trata de consolidar los puertos españoles como la conexión ideal para atraer no sólo las mercancías de import/export entre España y los principales centros neurálgicos de la economía europea, sino también como base para los tránsitos marítimos, particularmente los procedentes de otros continentes como África, América y Asia. 
España y sus puertos pueden seguir desempeñando un papel preponderante en el contexto del transporte mundial en el futuro, pero dependerá de cómo se afronte y cómo se adapten a los cambios que impondrá la revolución industrial 4.0. La privilegiada posición geográfica de España, emplazada en la intersección de las principales rutas del transporte marítimo internacional, y la rápida adaptación en los últimos años de sus infraestructuras marítimas a los nuevos requerimientos de la demanda mundial (con una capacidad instalada de mil millones de toneladas), sitúa a España en el Top 10 del ranking mundial en calidad de infraestructuras portuarias (Pereira Iglesias, 2018).

\section{F.3 Descarbonización de puertos}

Los puertos españoles ya han comenzado a aplicar bonificaciones del $50 \%$ en la tasa portuaria a aquellos buques que utilicen el gas natural licuado (GNL) como combustible o bien que utilicen gas en puerto para sus motores auxiliares o se conecten a la red eléctrica (Echazarreta y Costa, 2018). Además, se mejoran las infraestructuras y se modifican las regulaciones necesarias para permitir el avituallamiento de buques con combustibles alternativos en condiciones de mercado, y se bonifica la reducción de emisiones por encima de los límites actualmente establecidos, desarrollando también incentivos económico-fiscales sobre la demanda. La búsqueda de un puerto más sostenible llevará a mejorar la calidad ambiental y, en consecuencia, la calidad de vida de las personas y el medio ambiente.

\section{F.4 Open Data}

Consiste en la obtención de una mayor transparencia en la gestión portuaria a través de plataformas de apertura de datos públicos. Con el empleo de Open Data se consigue poner la información que posee el sector público, en este caso la Autoridad Portuaria, al alcance de todos de una manera estandarizada y abierta, en formato digital. De esta manera se permite a todos los ciudadanos y entidades tener acceso a datos sobre la administración, garantizando la transparencia, la eficiencia y la igualdad de oportunidades a la hora de crear valor.

\section{F.5 Prácticas logísticas más avanzadas y alta capacidad de solución}

Las terminales automatizadas permiten a los puertos manejar los contenedores de manera más eficiente planificando el almacenamiento de acuerdo con los tiempos de recolección y transbordo. Esto reduce los movimientos innecesarios y los tiempos de operación y, por tanto, aumenta la productividad. Por otro lado, mientras los seres humanos son susceptibles al cansancio o las distracciones, los sistemas automatizados operan siempre al mismo rendimiento sin importar imprevistos, de forma que cuentan con la ventaja de constantes, produciéndose rara vez algún fallo. Esto hace que el puerto sea más fiable en cuanto a demoras y aparición de imprevistos, los cuales muchas veces se deben a errores humanos que podrían haber sido evitados. Asimismo, también evita la incertidumbre que pueden ocasionar situaciones como las huelgas. Este aumento de fiabilidad y seguridad es percibido por los clientes, lo que se traduce en un aumento de la competitividad del puerto. La interconexión conseguida con la digitalización permite interactuar y analizar datos, prediciendo posibles errores en los procesos portuarios, al tiempo que permite reconfigurar los mismos y adaptarse a los cambios, obteniendo rendimientos operativos superiores. El empleo de prácticas logísticas más avanzadas y orientadas a las necesidades del mercado permite desarrollar servicios dedicados y de valor añadido con los que atraer y fidelizar a los clientes. Un ejemplo de ello es la mejora en la agilidad de los servicios aduaneros y de inspección o la reducción de las barreras de entradas para clientes del hinterland. 


\section{F.6 Reducción de costes de operación y mantenimiento}

La automatización de los sistemas del puerto conlleva la reducción de mano de obra, con lo que se reducen considerablemente los costes de las operaciones. Los sistemas automatizados sólo requieren de un software y un trabajador portuario especializado para su control, a diferencia de los sistemas tradicionales que demandan mucha mano de obra menos especializada. La automatización trae consigo el aumento de equipos eléctricos y la reducción de los equipos de combustión, lo que reduce los costes de mantenimiento y la probabilidad de accidente. La automatización también conlleva una reducción de daños en equipos y cargas, lo que contribuye a abaratar el mantenimiento.

\section{F.7 Incremento de la seguridad}

La automatización a nivel portuario incrementa la seguridad de las distintas terminales, pues mediante inteligencia artificial se controla mejor quién entra y quién sale del puerto. Todas las puertas y accesos son automáticos y se dispone de un sistema de entrada mediante reconocimiento óptico, huellas dactilares o por voz, que hacen el puerto mucho más seguro. Por otro lado, permite tener un mayor control de los procesos de carga $y$ descarga, al tiempo que se evita perder la documentación al estar todo digitalizado.

\section{Análisis externo}

En este caso particular, las amenazas y las oportunidades son caras opuestas de la misma moneda, no temas diferentes. Su definición se basó en un análisis externo de la situación portuaria española.

\section{Amenazas}

\section{A.1 Elementos políticos e institucionales}

El contexto de las políticas es importante para entender las iniciativas de los smart ports que comprende tanto elementos políticos como institucionales. La transformación hacia la digitalización exige la interfaz de los elementos tecnológicos con los elementos políticos e institucionales. Bajo la dimensión política, históricamente, las interacciones entre los sectores público y privado han sido bastante complicadas. Sin embargo, los distintos modos de transporte tienen que mantener una estrecha relación en aras de conseguir una digitalización global, implicando también elementos políticos e institucionales en el objetivo.

\section{A.2 Mala integración del resto de agentes del sector portuario}

El proceso de cambio hacia el nuevo sistema portuario y la modificación, e incluso sustitución, de las instalaciones pueden traer consigo una inicial lentitud e ineficacia en las operaciones del puerto. Esta circunstancia puede ser aprovechada por puertos competidores extranjeros, quedándose con parte de los clientes actuales de los puertos españoles. Las autoridades portuarias pueden sentirse amenazadas por la diferente implantación del sistema de digitalización en los diferentes puertos, puesto que no todos los puertos están igual de preparados para la digitalización, y una mejor y más rápida recepción de los nuevos procesos tecnológicos por parte de un puerto determinado puede hacer disminuir la atracción de clientes actual de otro puerto. Por otro lado, de nada sirve ser el único puerto con la tecnología e iniciativas de smart ports si el resto de puertos, buques y navieras, transportistas, comerciantes y vendedores no han implementado dichas actuaciones.

\section{A.3 Cambios de legislación y normativa}

La inconsistencia en las normas y regulaciones nacionales y regionales relacionadas con las políticas no pueden ayudar a ampliar las iniciativas. El empleo del Big Data 
y el uso masivo de datos tiene unas implicaciones legales que vienen controladas por las distintas administraciones de cada país, comunidad o región. Los cambios de legislación, normativa, derecho de protección de la intimidad, la imposibilidad de almacenar datos de carácter personal y los futuros cambios de legislación pueden hacer que esta transformación digital no sea tan útil como a priori se pensaba. Al final la utilidad de esta digitalización vendrá principalmente condicionada por las restricciones que ponga el almacenamiento y empleo de estos datos. De nada sirve que puertos y buques tengan mucha información si su uso no está permitido en el país de destino.

\section{A.4 Sistemas vulnerables a las amenazas cibernéticas}

La digitalización de nuestro nuevo mundo y el desarrollo y progreso de las tecnologías pone de manifiesto una mayor vulnerabilidad y sensibilidad a ataques a través del ciberespacio. Actualmente el Big Data existe y es una nube intangible albergada en el aire y a disposición de todos los habitantes del mundo. Esto puede generar grandes problemas, ya que los datos privados (ya sean personales, protegidos, gestionados por administraciones, etc.) son el motor del siglo XXI al ser muy valiosos para las empresas. La dependencia de la tecnología, tanto en los puertos como en los buques, es cada vez mayor. Así, la tripulación se va reduciendo a medida que se utilizan sistemas informáticos para la navegación, al tiempo que la descarga es más rápida y es posible realizar un seguimiento de mercancías en los puertos. Desafortunadamente, estos sistemas también son muy vulnerables a las amenazas cibernéticas.

\section{A.5 Asimetría con otros modos de transporte}

Como cabe esperar, la digitalización no está llegando únicamente al transporte marítimo, de forma que el transporte terrestre y el aéreo están avanzando cada día. Es en el transporte de personas dónde se le presenta una mayor competencia al transporte marítimo puesto que en el transporte de mercancía, no dejan de luchar el ferrocarril y el avión por abrirse paso. Sin embargo, no cabe duda que para una correcta digitalización del transporte es necesario una colaboración y participación de todos los modos que formen parte de la cadena. El ahorro de costes, una mayor agilidad en toda la cadena de suministro y una mejora en la eficiencia operacional son las principales ventajas que el sector logístico ve en la digitalización de los documentos relacionados con el transporte de mercancías, que afectan a todos los modos de transporte.

\section{A.6 Incertidumbre financiera internacional}

Existe una falta de presupuesto y de recursos debido a que las finanzas públicas agotadas están desacelerando las inversiones públicas. Además, faltan metodologías y métricas apropiadas y sistemáticas para informar y verificar los rendimientos de las inversiones. La situación financiera, la falta de créditos y las regulaciones sobre las instituciones financieras para reducir la exposición al riesgo mediante la creación de bases de depósitos más sólidas están limitando los flujos de efectivo disponibles. Esto provoca una ralentización de las inversiones en actuaciones de digitalización de los puertos.

\section{Oportunidades}

Las oportunidades son áreas potenciales en las que el puerto puede identificar el potencial de crecimiento, ganancias y participación de mercado. Por lo tanto, se trata de un punto de posible desarrollo positivo que afecta de manera externa al puerto. 


\section{O.1 Nuevas líneas de negocio en mercados en desarrollo}

El crecimiento del comercio internacional en un mundo globalizado abre una oportunidad de negocio para los puertos al abrir nuevas líneas de negocio en mercados de países en vías de desarrollo. Las economías de estos países se encuentran en auge y demandan cada vez más productos de alto nivel y precio (productos tecnológicos, joyas, coches deportivos, armamento avanzado, etc.) y no sólo de primera necesidad como venía sucediendo. Además, la digitalización permite gestionar de manera más eficiente las mercancías y los pasajeros, consiguiéndose transportar más volumen de manera conjunta, viéndose favorecido por el factor de escala y mejorando aún más la competencia.

\section{O.2 Madurez tecnológica}

La productividad industrial se está mejorando, lo que permite a los actores involucrados dotar de una amplia gama de productos y servicios a los puertos. Esto puede ayudarles a aventurarse significativamente en productos y servicios con un alto grado de inmediatez.

\section{O.3 Creación de oportunidades de trabajo}

La conveniencia de poder trabajar desde casa podría ir acompañada de un control continuo. La esperanza de poder trabajar menos podría verse sofocada por la posibilidad de trabajar en cualquier lugar y en cualquier momento. Los nuevos empleos especializados aumentarán la competencia y, en consecuencia, disminuirá las ganancias de los individuos. Lo que sí es seguro, es que las empresas que mantengan el ritmo sólo tienen que ganar en esta era de innovaciones. Pero algunos de estos empleos perdidos pueden ser reemplazados por la necesidad de personal empleado en el manejo del nuevo equipo tecnológico. Finalmente, varios estudios muestran que, en el futuro, la mayoría del transporte pasará a través de puertos y aeropuertos. Durante la construcción de puertos sostenibles, la creación de oportunidades de trabajo, el desarrollo de la fuerza laboral y el espíritu empresarial generarán una mejora significativa en la productividad y también establecerán un alto grado de competitividad económica.

\section{O.4 Sinergias puerto ciudad}

La digitalización de los puertos en relación con el desarrollo de las smart cities permite una interacción sinérgica entre ambas instituciones, sus gobernantes y sus ciudadanos. Los puertos digitales son por y para las personas, siendo, por tanto, el agente clave en su futuro desarrollo. Las plataformas digitales permiten que el ciudadano pueda conocer las oportunidades que le ofrece el puerto, permitiendo también la creación de riqueza que el puerto supone para el conjunto de la población. De la misma manera, el ciudadano puede trasladar sugerencias o propuestas a la dirección del puerto que se traduzcan en mejoras para el día a día de la ciudadanía, fomentando una mejor convivencia entre el puerto y la ciudad.

\section{O.5 Cadena de suministro eficiente y predictiva}

Una gestión más sostenible permite reducir la contaminación, ganando flexibilidad y agilidad. Los puertos inteligentes reducen los costes del transporte y, consecuentemente, sus conexiones son mucho más atractivas, ofreciendo una ventaja competitiva muy importante respecto a los otros enclaves. En este contexto, la tecnología y la innovación se convierten en elementos clave para facilitar información en tiempo real, prestando nuevos servicios a partir del flujo de datos y digitalizando procesos. La integración con la ciudad y con el hinterland-foreland reduciría las colas de camiones y atascos. 
Para hacer frente a ellos, sería clave que se compartiese, fusionasen y analizaran los datos disponibles con vistas a optimizar los procesos y el rendimiento, para lo que el Big Data sería primordial.

\section{O.6 Inserción en la cadena global de España en la red chainPORT}

Esta red tiene como objetivo establecer una cadena global de puertos inteligentes líderes en el desarrollo e implementación de soluciones tecnológicas para compartir conocimiento, crear una base para el desarrollo conjunto de soluciones, innovadoras $\mathrm{y}$ orientadas al futuro, y desarrollar estrategias comunes para el sector portuario.

\section{Dafo cruzada}

Con los datos analizados, es necesario reforzar las fortalezas y reducir las debilidades, explotando el conocimiento de amenazas y oportunidades.

\section{Fortalezas + Oportunidades}

Gracias a su posición, España puede convertirse en una potencia mundial, como es Gran Bretaña o Estados Unidos. El español es el tercer idioma más hablado en el mundo, el segundo si no se considera el chino, el cual es podo hablado fuera de China. Por otro lado, la Península Ibérica domina tanto el mar Mediterráneo como los océanos más importantes, que la proyectan en una posición dominante en un futuro regentado por las infraestructuras y la tecnología de transporte. Los puertos españoles aún no se encuentran en el nivel de sostenibilidad del puerto de Antwerp (Bélgica) o del puerto de Hamburgo (Alemania). Sin embargo, España tiene muchos más recursos y es normal que mantenga un nivel de avance más lento que los países con menos estructuras.

\section{Fortalezas + Amenazas}

Con una planificación previa, es posible volver a capacitar a la masa laboral para no tener que reducirla. Además, reducir la presencia humana en beneficio de las máquinas inteligentes es precisamente uno de los errores sobre los científicos e investigadores advierten, puesto que la inteligencia artificial (IA) debe ayudar al ser humano y no reemplazarlo. Además, en este nuevo mundo inteligente, para preservarse de las decisiones de los países aislacionistas, España podría formar su propia comunidad con sus antiguas colonias puesto que los nuevos técnicos especializados pueden derribar muros y viejos conflictos pasados.

\section{Debilidades + Oportunidades}

Será imposible aprovechar al máximo las oportunidades que ofrece la posición actual y la tecnología, a menos que se redacte un reglamento único que permita ayudar a los distintos organismos a cooperar entre sí. Además, es muy importante garantizar que las personas crezcan junto con el país (tanto a los estudiantes como profesionales ya activos en su sector). En cuanto a las emisiones, España avanza por el camino correcto.

\section{Debilidades + Amenazas}

La inadecuación de los operadores actuales, junto con la reducción de la voz de los sindicatos, podría propiciar una pérdida o un reemplazo de la masa laboral. Para esto, es necesario discutir el problema y planificar una solución, siendo la máxima preocupación que las personas que no perciban la digitalización se queden atrás. Del mismo modo que para crear una ciudad inteligente, la ciudad debe crecer junto con su infraestructura; los ciudadanos, y especialmente los trabajadores, deben integrarse 
en este nuevo proyecto. Es necesario proporcionar cursos de actualización y tener siempre un plan B puesto que la peor amenaza es la que no se prevé pues bloquea todo el sistema.

\section{Conclusiones}

La adopción de sistemas y tecnologías de la información (IT/IS) ha allanado repetidamente el camino para la modernización de los puertos marítimos. A pesar de la creciente importancia de los sistemas de información para resolver los desafíos actuales y futuros, se ha prestado poca atención a este tema en el área de logística marítima.

La marcha de la digitalización continúa cambiando los procesos logísticos en los puertos marítimos modernos. Sin embargo, el éxito de la transformación digital radica no sólo en la adopción de tecnologías modernas y métodos avanzados, sino principalmente en la adaptación de estructuras organizativas. En este sentido, se aprecia que las iniciativas digitales, a pesar de ser innovadoras y con un propósito específico, pueden fallar fácilmente si los diferentes requisitos, perspectivas e implicaciones para los actores individuales no se consideran adecuadamente, especialmente en ecosistemas complejos como son los puertos marítimos. La alineación de las estrategias y la cooperación entre las partes interesadas juegan un papel crucial en la transformación de las operaciones portuarias con objetivos comunes. En este sentido, se debe considerar las perspectivas intra, inter y metaorganizacionales para analizar las opciones, así como los costes y beneficios resultantes de las transformaciones digitales. Para construir y evaluar diferentes formas de cooperación, se observa que se necesita más investigación, especialmente cuantitativa.

En un horizonte cercano, los puertos buscan la plena automatización de sus procesos, la gestión del riesgo operacional y la sincronomía, en un marco de economía colaborativa.

Las claves para obtener la máxima eficiencia a través de la digitalización van a sustentarse en un cambio cultural y una nueva visión del sector portuario frente a la innovación (Figura 4).

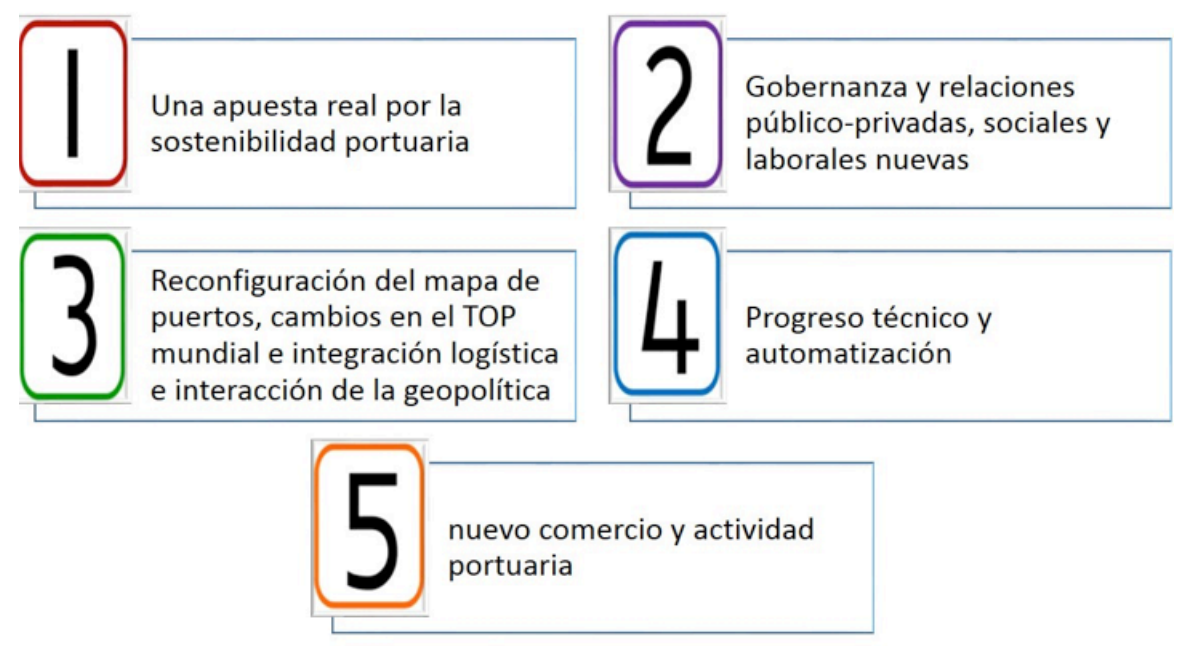

Figura 4. Claves para obtener la máxima eficiencia. Fuente: Elaboración propia. 
En la Figura 5 se muestra un mapa de perspectiva global a corto y medio plazo con los elementos más destacados por los expertos, diferenciando entre los elementos que pongan innovación en valor y los que suponen tan solo imitación en valor.

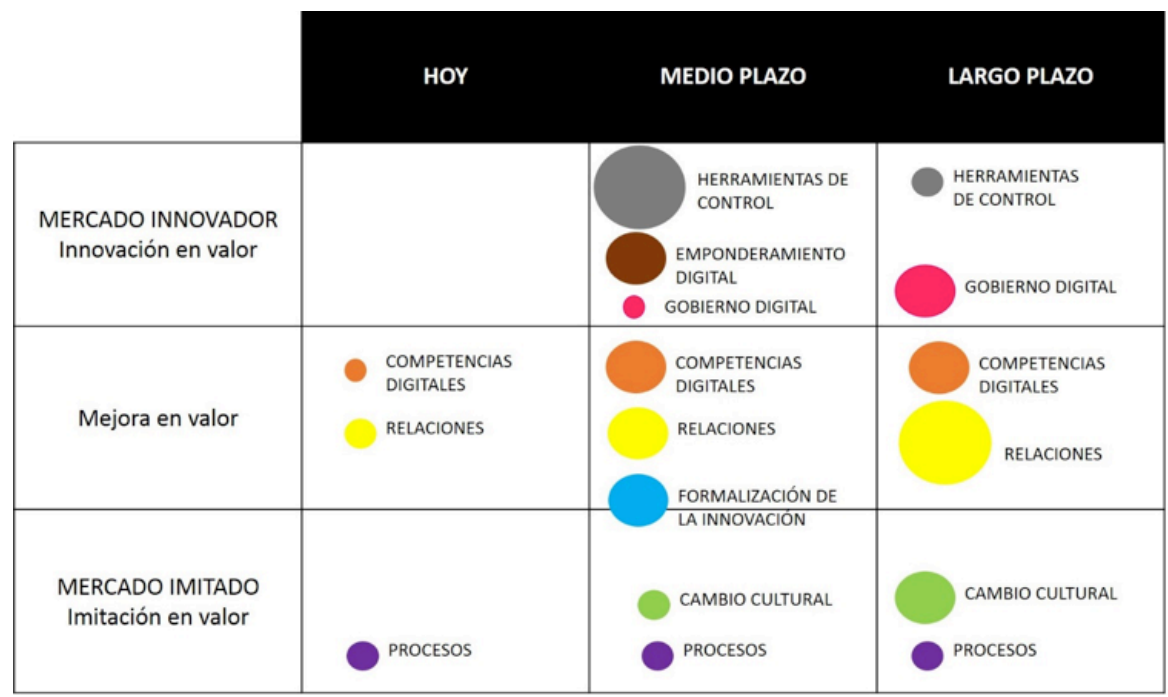

Figura 5. Mapa de perspectiva global. Fuente: Elaboración propia.

Los expertos notaron especial incidencia en el futuro de la digitalización en los aspectos relativos al cambio de paradigma que va suponer este proceso en los puertos, en la transparencia en el proceso y en los datos, los cuales van a marcar los pasos siguientes teniendo que poner especial atención en la ciberseguridad. 


\section{Q Bibliografia}

» Arguedas, V. F.; Pallotta, G. y Vespe, M. (2017). Maritime Traffic Networks: From historical positioning data to unsuper-vised maritime traffic monitoring. IEEE Transactions on Intelligent Transportation Systems, 19(3), 722-732.

» Brouer, B. D.; Karsten, C. V. y Pisinger, D. (2016). Big data optimization in maritime logistics. In Big data optimization: Recent developments and challenges (pp. 319-344). Springer, Cham.

"Cervantes, J. G.; Jerez, A. R.; Fierro, Á.; Ocaña, P. y Rivero, A. (2013). Plataforma tecnológica para la mejora de la sostenibilidad, la medición y la relación con el entorno en territorios. Escenarios: empresa y territorio, 2(2), 17-36.

»Cimino, M. G. C. A.; Palumbo, F.; Vaglini, G.; Ferro, E.; Celandroni N. y La Rosa, D. (2016). Evaluating the impact of smart technologies on harbor's logistics via BPMN modeling and simulation. Inf Technol Manag. doi: 10.1007/s10799-016-0266-4

»Davarzani, H.; Fahimnia, B.; Bell, M. y Sarkis, J. (2016). Greening ports and maritime logistics: A review. Transportation Research Part D: Transport and Environment, 48, 473-487.

"Echazarreta, C. y Costa, A. (2018). Sustainable Companies, Addressing the Climate Change: A Theoretical Review. Global Journal of Management and Business Research.

»Fruth, M. y Teuteberg, F. (2017). Digitization in maritime logistics-What is there and what is missing?. Cogent Business \& Management, vol. 4, no 1, p. 1411066.

" Grasso, M.; Pagola, L. y Zanotti, A. (2017). Políticas de inclusión digital en Argentina. Usos y apropiaciones dentro y fuera de la escuela. Pixel-Bit. Revista de Medios y Educación, núm. 50, pp. 95-107.

» Haraldson, S. (2015). Digitalization of sea transports-Enabling sustainable multi-modal transports.

» Heilig, L. y VOß, S. (2017a). Information systems in seaports: a categorization and overview. Information Technology and Management, 18(3), 179-201.

» Heilig, L. y VOß, S. (2017b). Status quo and innovative approaches for maritime logistics in the age of digitalization: a guest editors' introduction.

" Lee, H.; Aydin, N.; Choi, Y.; Lekhavat, S. y Irani, Z. (2018). A decision support system for vessel speed decision in mari-time logistics using weather archive big data. Computers y Operations Research, 98, 330-342.

»Ministerio de Fomento. (2017). La política europea de transporte. En Ministerio de Fomento. Transporte Intermodal. Disponible en https://www.fomento.gob. es/MFOM/LANG_CASTELLANO/DIRECCIONES_GENERALES/TRANSPORTE_ TERRESTRE/TRANSPORTE_INTERMODAL/

»Panayides, P. M. y Song, D. (2013) Maritime logistics as an emerging discipline. Maritime Policy Manag 40(3):295-308.

»Pereira Iglesias, S. (2018). Análisis de los accesos por ferrocarril a los principales puertos españoles y propuestas de actuación (Master's thesis, Universitat Politècnica de Catalunya).

"Sánchez, R. y Barleta, E. (2018). Reflexiones sobre el futuro de los puertos de contenedores por el nuevo comportamiento de la contenedorización. 
»Sánchez, R. y Mouftier, L. (2016). Reflexiones sobre el futuro de los puertos: del estrés actual al cambio y la innovación del futuro.

"Sánchez, R.; Palma Barleta, E. y Mouftier, L. (2017). Reflexiones sobre el futuro de los puertos de contenedores.

"Zaman, I.; Pazouki, K.; Norman, R.; Younessi, S. y Coleman, S. (2017). Challenges and opportunities of big data analytics for upcoming regulations and future transformation of the shipping industry. Procedia engineering, 194, 537-544.

\section{Nicoletta González-Cancelas / nicoleta.gcancelas@upm.es}

Doctora Ingeniera de Caminos, Canales y Puertos por la Universidad Politécnica de Madrid. Profesor Contratado Doctor del Departamento de Ingeniería Civil, Transportes de la Universidad Politécnica de Madrid. Es especialista en Logística y transporte. Pertenece al Grupo de Investigación reconocido de la Universidad Politécnica de Madrid y es responsable de las líneas de investigación en Logística y Data Mining aplicado al transporte.

\section{Beatriz Molina Serrano / beatriz.molinas@alumnos.upm.es}

Doctora Ingeniera de Caminos, Canales y Puertos por la Universidad Politécnica de Madrid. Investigadora de la Escuela Técnica Superior de Ingenieros de Caminos, Canales y Puertos de la Universidad Politécnica de Madrid. Jefe de proyecto en la empresa de ingeniería PROINTEC, S.A.U. Es especialista en transporte y seguridad vial.

\section{Magdalena Esteban-Infantes / magdalena.estebanic@gmail.com}

Ingeniera de Caminos, Canales y Puertos por la Universidad Politécnica de Madrid. Máster en Gestión y Planifación Portuaria e Intermodalidad.

\section{Francisco Soler-Flores / francisco.soler@unir.net}

Licenciado en Matemáticas por la Universidad de Almería. Doctor en Tecnología y Sistemas de Información por la Universidad de Castilla-La Mancha. Profesor en las áreas de Estadística, Informática e Inteligencia Artificial en la Universidad Internacional de la Rioja y en la Universidad de Alcalá, ha sido investigador y profesor en grado, master y cursos de especialización en diferentes universidades públicas y privadas como la Universidad Politécnica de Madrid o la Universidad Europea

\section{Alberto Camarero-Orive / alberto.camarero@upm.es}

Doctor Ingeniero de Caminos, Canales y Puertos por la Universidad Politécnica de Madrid y Licenciado en Economía y en Administración, y Dirección de Empresas por la Facultad de Ciencias Sociales y Jurídicas de la Universidad Carlos III de Madrid. Universidad. Profesor titular del Departamento de Ingeniería Civil, Transporte y Urbanismo de la Universidad Politécnica de Madrid. Es especialista en Logística y Transporte. 